OPEN ACCESS

Edited by:

Fanghua Liu,

Yantai Institute of Coastal Zone

Research (CAS), China

Reviewed by:

Pier-Luc Tremblay,

Wuhan University of Technology,

China

Yong Yuan,

Guangdong University of Technology,

China

*Correspondence:

Xin Wang

xinwang1@nankai.edu.cn

Specialty section:

This article was submitted to Microbiotechnology, Ecotoxicology and Bioremediation,

a section of the journal

Frontiers in Microbiology

Received: 13 April 2018

Accepted: 25 June 2018

Published: 17 July 2018

Citation:

Wan Y, Zhou L, Wang S, Liao C, Li N, Liu W and Wang X (2018) Syntrophic Growth of Geobacter sulfurreducens Accelerates Anaerobic Denitrification.

Front. Microbiol. 9:1572

doi: 10.3389/fmicb.2018.01572

\section{Syntrophic Growth of Geobacter sulfurreducens Accelerates Anaerobic Denitrification}

\author{
Yuxuan Wan', Lean Zhou' ${ }^{1}$, Shu Wang ${ }^{2}$, Chengmei Liao', Nan Li², Weitao Liu' and \\ Xin Wang ${ }^{1 *}$
}

${ }^{1}$ MOE Key Laboratory of Pollution Processes and Environmental Criteria, Tianjin Key Laboratory of Environmental Remediation and Pollution Control, Nankai University, Tianiin, China, ${ }^{2}$ School of Environmental Science and Engineering, Tianjin University, Tianjin, China

Nitrate is considered as a contamination since it's over discharging to water incurs environmental problems. However, nitrate is an ideal electron sink for anaerobic pollutant degraders desiring electron acceptors due to the high redox potential. Unfortunately, not all degraders can directly reduce nitrate, and the anaerobic direct interspecies electron transfer (DIET) between degraders and denitrifiers has not been confirmed yet. Here we demonstrated that syntrophic growth of Geobacter sulfurreducens PCA with denitrifying microbial community at anaerobic condition eliminated the lag phase of $15 \mathrm{~h}$ and improved the denitrification rate by $13 \sim 51 \%$ over a broad C/N ratio of 0.5 to 9 . Quantitative PCR revealed that $G$. sulfurreducens selectively enhanced the expression of nirS coding for a cytochrome cd1-nitrite reductase, resulting in a fast and more complete denitrification. Geobacter also selectively enriched its potential denitrifying partners Diaphorobacter, Delftia, and Shinella - to form spherical aggregates. More studies of the binary culture system need to be carried out to confirm the syntrophic mechanism of Geobacter and denitrifiers in the future. These findings extend our knowledge on understanding the anaerobic bacterial interspecies electron transfer in the denitrification process, which has broader implications in fast selection and stabilization of denitrifiers in wastewater treatment plant, and general understanding of ecology for nitrogen and metal cycling.

Keywords: anaerobic denitrification, syntrophic growth, aggregation, Geobacter sulfurreducens, interspecies electron transfer

\section{INTRODUCTION}

Interspecies electron transfer is critical in anaerobic digester due to the lack of electron acceptors. Syntrophic partners usually grow together to overcome energy barriers and share metabolic electrons during complex organic pollution degradation (Boone et al., 1989; Jackson and McInerney, 2002; Ishii et al., 2005). Microorganisms grown in syntrophic community transfer electrons via a few simple substrates including $\mathrm{H}_{2}$, formate, methanol, and acetate (Stams and Plugge, 2009). Recently, the direct interspecies electron transfer (DIET), an new electron transfer mode of microorganisms initially demonstrated in cocultures of Geobacter species (Rotaru et al., 2014; Lovley, 2017), is increasingly being recognized as an important way of sharing 
electrons when exoelectrogens are found in syntrophic community although the details and exact mechanisms still need further studies. Electrons were demonstrated to transfer directly from Geobacter metallireducens to Geobacter sulfurreducens in cocultures with ethanol as the electron donor and fumarate as the electron acceptor (Summers et al., 2010). Studies with mutant strains of G. sulfurreducens, as well as genome-wide transcriptomic analysis, demonstrated that $\mathrm{H}_{2}$ or formate is not their interspecies electron carrier. However, Pseudomonas aeruginosa prefer to transfer electrons to G. sulfurreducens via hydrogen or formate (Rotaru et al., 2012; Semenec et al., 2018). The above mentioned syntrophic partners often gather together to form aggregates to minimize the distance of adjacent cells to improve mass transfer or electron transfer.

Nitrate is a natural electron acceptor in wastewater with high redox potential of $+0.433 \mathrm{~V}$ to nitrite versus standard hydrogen electrode. The use of electrons from organic pollutants to reduce nitrate has a great value in terms of wastewater treatment. However, not all microorganisms are able to reduce nitrate to produce adenosine triphosphate (ATP) for their growth. Only bacteria namely denitrifiers with nitrate reductase (NAR), nitrite reductase (NIR), nitric oxide reductase (NOR), or nitrous oxide reductase $\left(\mathrm{N}_{2} \mathrm{OR}\right)$ are able to reduce nitrate and release molecular nitrogen $\left(\mathrm{N}_{2}\right)$ or nitrous oxide $\left(\mathrm{N}_{2} \mathrm{O}\right)$ (Zumft, 1997). G. sulfurreducens grown on acetate was not able to reduce nitrate. It had been demonstrated that the syntrophic growth of G. sulfurreducens and Wolinella succinogenes (not use acetate) can oxidize acetate and reduce nitrate to ammonium efficiently. The electrons from acetate oxidation was transferred probably through interspecies hydrogen transfer $(0.02$ to $0.04 \mathrm{~Pa})$ to $W$. succinogenes for nitrate reduction (Cord-Ruwish et al., 1998). However, this process is called "dissimilatory nitrate reduction to ammonium (DNRA)," which is different from the denitrification with $\mathrm{N}_{2}$ or $\mathrm{N}_{2} \mathrm{O}$ as the end product. So far, interspecies electron transfer between Geobacter and denitrifier was only found between $G$. sulfurreducens and an autotrophic Thiobacillus denitrificans when conductive nano$\mathrm{Fe}_{3} \mathrm{O}_{4}$ was added (Kato et al., 2012). Unfortunately, this coculture seems to experience a DNRA process with ammonium as the end product. Nitrate cannot be reduced when $\mathrm{Fe}_{3} \mathrm{O}_{4}$ was removed, indicating that the denitrification between Geobacter and denitrifier is still not confirmed. Our hypothesis is that the denitrification through interspecies electron transfer may play a key role in mixed community when the growing condition was significantly changed, because the interspecies electron transfer, either through interspecies hydrogen transfer or DIET, is believed as an efficient route for different species to balance energy (Lovley, 2017).

Here G. sulfurreducens PCA was added into a denitrifying microbial community when the oxygen concentration and the carbon source were simultaneously changed. Pink aggregates were observed in cocultures, indicating the syntrophic growth. The mechanism was further explored according to the nitrate and nitrite removal, reverse transcription quantitative PCR and illumina sequencings. Denitrification at different $\mathrm{C} / \mathrm{N}$ ratio was also investigated using this syntrophic community.

\section{MATERIALS AND METHODS}

\section{Microbial Growth and the Experimental Design}

Denitrifying microbial community was acclimated from activated sludge collected from beer brewery wastewater treatment plant (Snowflake Brewery Co., Ltd., Tianjin, China) with methanol as the sole carbon source. The basal medium used for acclimation contained: $\mathrm{Na}_{2} \mathrm{HPO}_{4} 0.8 \mathrm{~g} / \mathrm{L}, \mathrm{CaCl}_{2} 0.1 \mathrm{~g} / \mathrm{L}, \quad \mathrm{FeSO}_{4} \cdot 7 \mathrm{H}_{2} \mathrm{O}$ $0.07 \mathrm{~g} / \mathrm{L}$, and $\mathrm{MgSO}_{4} \cdot 7 \mathrm{H}_{2} \mathrm{O} 0.1 \mathrm{~g} / \mathrm{L}$. $20 \mathrm{mM}$ methanol and 3 9 $\mathrm{mM}$ nitrate were supplemented as the electron donor and acceptor. Activated sludge was acclimated at anoxic under shaking conditions $(150 \mathrm{rpm})$ in the growth medium at $30^{\circ} \mathrm{C}$. The medium was refreshed every 2 days $(10 \% \mathrm{v} / \mathrm{v})$ until the denitrification performance was stable (Supplementary Figure S1). The whole acclimation required 18 days. This community was then consecutively transferred for 3 batches before stored at $-80^{\circ} \mathrm{C}$ with phosphate buffer and glycerin. In order to ensure the reproducibility of each tests, this denitrifying microbial community was reactivated from the same batch of frozen stock. The strain G. sulfurreducens PCA (ATCC 51573) was from laboratory frozen stocks, which was incubated in sterile anaerobic bottles (100 $\mathrm{mL}$ in capacity) before use.

Both the denitrifying microbial community $(70 \mathrm{~mL}$, $\left.\mathrm{OD}_{600}=0.32\right)$ and $G$. sulfurreducens PCA cells $(100 \mathrm{~mL}$, $\mathrm{OD}_{600}=0.2$ ) were collected at logarithmic growth phase by centrifugation (4000 rpm, $5 \mathrm{~min}$ ) and washed twice to resuspend to $70 \mathrm{~mL}$ of anaerobic sterile medium to reach an $\mathrm{OD}_{600}$ of 0.6 (marked as group G-D). The medium (per liter of distilled water) contains $0.1 \mathrm{~g} \mathrm{KCl}, 1.5 \mathrm{~g} \mathrm{NH}_{4} \mathrm{Cl}, 0.69 \mathrm{~g} \mathrm{NaH}_{2} \mathrm{PO}_{4} \cdot 2 \mathrm{H}_{2} \mathrm{O}$, $0.075 \mathrm{~g} \mathrm{CaCl}_{2}, 0.1 \mathrm{~g} \mathrm{MgCl}_{2} \cdot 6 \mathrm{H}_{2} \mathrm{O}, 5 \mathrm{~mL}$ vitamin solution, and $12.5 \mathrm{~mL}$ trace minerals (Lovley and Phillips, 1988). $13 \mathrm{mM}$ acetate and $3.7 \mathrm{mM}$ nitrate $(6 \mathrm{mg} \mathrm{C} / \mathrm{mg} \mathrm{N}$ to offer enough carbon for denitrification) were added as the electron donor and acceptor. It was flushed using $\mathrm{N}_{2} / \mathrm{CO}_{2}(80: 20)$ gas for $30 \mathrm{~min}$ to remove oxygen, sealed with butyl rubber septa and aluminum cover, then sterilized at $121^{\circ} \mathrm{C}$ for $20 \mathrm{~min}$. Denitrifying bacteria (marked as Group D) and G. sulfurreducens PCA (marked as Group $G$ ) were individually inoculated to the same anaerobic medium $\left(\mathrm{OD}_{600}=0.6\right)$ as the controls. In order to confirm that the changes of denitrification performance were due to the living $G$. sulfurreducens PCA but not its intercellular or extracellular components, G. sulfurreducens PCA inactivated by ultraviolet radiation $(10 \mathrm{~h})$ or ultrasonic fragmentation (300 W, $20 \mathrm{~min}$ ) was also added with denitrifying bacteria into anaerobic medium (Table 1 ). For the $\mathrm{C} / \mathrm{N}$ ratio tests, denitrifying bacteria and denitrifying bacteria $+G$. sulfurreducens PCA were parallelly grown at $\mathrm{C} / \mathrm{N}$ of $0.5,1,3,6$, and 9 . All these experiments were carried out in a glove box (1029, Thermo Scientific, United States) in triplicate.

\section{Nitrogen, Acetate Analysis and Aggregates Imaging}

Samples were taken from each anaerobic bottle with sterile syringes at different time points, and then filtrated through $0.22 \mu \mathrm{m}$ filter before the measurement of ammonium, 
TABLE 1 | The list of experimental groups.

\begin{tabular}{llllll}
\hline Group & D & G & G-D & G-D 1 & G-D 2 \\
\hline Denitrifiers & + & - & + & + & + \\
Geobacter & - & + & + & + (UV sterilization) & + (Ultrasonic fragmentation)
\end{tabular}

"+" and "-" denote the presence and absence of Geobacter sulfurreducens PCA or denitrifying microbial community.

nitrate, and nitrite. Nitrate and ammonium concentrations were measured by UV spectrophotometry and Nessler reagent spectrophotometry, respectively, using a UV-Vis spectrophotometer (T6, Purkinje General Instrument Co., Ltd., China) (Thomas and Cerdà, 2007). Nitrite was tested by Griess reagent spectrophotometry using multimode microplate reader (SPARK 10M, TECAN, Switzerland). Acetate was measured by high performance liquid chromatography (1525, Waters, China).

Aggregates were carefully collected from the anaerobic bottles. They were fixed with $2.5 \%$ (wt/vol) glutaraldehyde on the glass plate, and then dehydrated using a gradually increased ethanol solution from 50 to $100 \%$. The dried samples were coated with gold, and imaged using scanning electron microscope (SEM, Shimadzu SS-550, Japan).

\section{Molecular Microbial Analysis}

Cell density $\left(\mathrm{OD}_{600}\right)$ was measured by multimode microplate reader (SPARK 10M, TECAN, Switzerland) based on the absorbance at $600 \mathrm{~nm}$. The content of protein was measured using a BCA protein assay kit (Solarbio, Beijing).

Genomic DNA was extracted at $0,12,24$, and $48 \mathrm{~h}$ from the culture of group D and G-D (6 $\mathrm{mg} \mathrm{C} / \mathrm{mg} \mathrm{N})$ using the Soil Genomic DNA Kit (CW2091S, ComWin Biotech Co., Ltd., China) according to the standard protocol (Feng et al., 2016). Genomic DNA of the aggregates was also extracted for analysis. The extracted DNA was sent to Majorbio (Shanghai, China) for PCR amplification. Sequencing targeted at the hypervariable V3, $\mathrm{V} 4$ region of $16 \mathrm{~S}$ rDNA with the universal primers $338 \mathrm{~F}$ and $806 \mathrm{R}$, and the amplicons were subsequently determined on the Illumina MiSeq platform. R language was used for visualization of the results.

The activity of denitrifying genes (nirK and nirS) were measured using nitrite-reductase specific primers. The 8761040 and R3cd-Cd3af primer pairs (Supplementary Table S1) were used to assess the nirK and nirS activity (Henry et al., 2004; Throback et al., 2004; Srinivasan and Butler, 2017). 16S rRNA gene was chosen as the reference for making relative quantification. The primer pair used for $16 \mathrm{~S}$ rRNA gene quantification was $338 \mathrm{~F}$ and $806 \mathrm{R}$. The sequence of primers was listed in Supporting Information. RNA were extracted from the culture using QIAamp RNA Blood Mini Kit (52304, QIAGEN, Shanghai, China) and treated to remove any DNA contamination. The extracted RNA were then quantified with a microplate reader (NANO Quant infinite M200PRO, Tecan, Switzerland). mRNA was then converted into cDNA and stored at $-20^{\circ} \mathrm{C}$. Amplification of cDNA templates was carried out with Real Time PCR System (ViiA 7, Applied Biosystems, China) using SYBR Green as detection system in a reaction mixture of $16 \mu \mathrm{L}$ containing: $0.2 \mu \mathrm{L}$ of each primer, $8 \mu \mathrm{L}$ of $2 \times$ SYBR Green PCR master mix, $1 \mu \mathrm{L}$ of the template cDNA, and RNase-free water to make up to $16 \mu \mathrm{L}$. All primer pairs amplifying gene fragments were then run with an initial denaturation of the DNA at $95^{\circ} \mathrm{C}$ for $2 \mathrm{~min}$, followed by $40 \mathrm{cycles}$ of $10 \mathrm{~s}$ at $94^{\circ} \mathrm{C}, 10 \mathrm{~s}$ at $60^{\circ} \mathrm{C}$, and $40 \mathrm{~s}$ at $72^{\circ} \mathrm{C}$. Triplicate wells were run for each sample for each gene target. Melting curves and negative controls were run for each qPCR run. The relative quantities of nirK and nirS transcripts were logarithmic (base 2) fold changes comparing to the value at time $0 \mathrm{~h}$.

\section{RESULTS AND DISCUSSION}

\section{Denitrification Performance}

A lag period of $15 \mathrm{~h}$ was observed on nitrate reduction when the anoxic denitrifying microbial community was transferred to anaerobic condition with the simultaneous carbon source changing from methanol to acetate (Group D, Figure 1A). Nitrite, the intermediate product during denitrification, was not detected to increase at this period. The $59 \pm 16 \mu \mathrm{M}$ of nitrite at the beginning was believed to be the background value desorbed from the biomass (Figure 1B). After that, a linear decrease of nitrate was observed, with a nitrate removal rate of $30 \mathrm{~g} \mathrm{~N} / \mathrm{m}^{3} \cdot \mathrm{d}$. Nitrite slightly accumulated at the same time, which reached the maximum of $294 \pm 24 \mu \mathrm{M}$ at $23 \mathrm{~h}$.

Very interestingly, when G. sulfurreducens PCA was mixed with the denitrifying microbial community (Group G-D), nitrate reduction started immediately without any lag period. An adaption period is usually needed for the switch from anoxic to anaerobic growth and also from methanol as electron donor, as showed in Figure 1A. Geobacter adapted very well with the "new" niche, however, lacking of electron donor. It is hypothesized that Geobacter and several denitrifiers fast united as a strategy to survive through interspecies electron transfer, so that the lag phase seems to be eliminated. The denitrification rate was accelerated by $33 \%$ to $40 \mathrm{~g} \mathrm{~N} / \mathrm{m}^{3} \cdot \mathrm{d}$, and the peak nitrite concentration appeared $6 \mathrm{~h}$ earlier with a $27 \%$ lower value $(215 \pm 20 \mu \mathrm{M}$, Figure 1B). The nitrite concentration peak gradually decreased to the background in $24 \mathrm{~h}$ in Group D, but it was suddenly dropped down in Group G-D after the peak value was achieved at $17 \mathrm{~h}$, indicating that the denitrification of Group G-D was more complete than Group D. Both Groups D and G-D reached the maximum nitrate removal efficiency of $92 \%$, however, with different times. The addition of G. sulfurreducens PCA shortened the time needed to reach $90 \%$ nitrate removal by $22 \%$ from 54 to $42 \mathrm{~h}$. We did not find any $\mathrm{N}_{2} \mathrm{O}$ in the headspace, probably because $\mathrm{N}_{2} \mathrm{O}$ was usually produced at anoxic but not anaerobic condition (Ding et al., 2016; Yan et al., 2016). The concentration of ammonium was almost constant Group D and G, but slightly increased in Group G-D (Supplementary Figure S3), and acetate consumption matched the nitrate reduction (Supplementary Figure S4). Around 38\% of nitrate was reduced to ammonium, which confirmed that the denitrification but not DNRA process dominated in this system. This is very different from previous G. sulfurreducens PCA involved DNRA reports (Cord-Ruwish et al., 1998; Kato et al., 2012). 

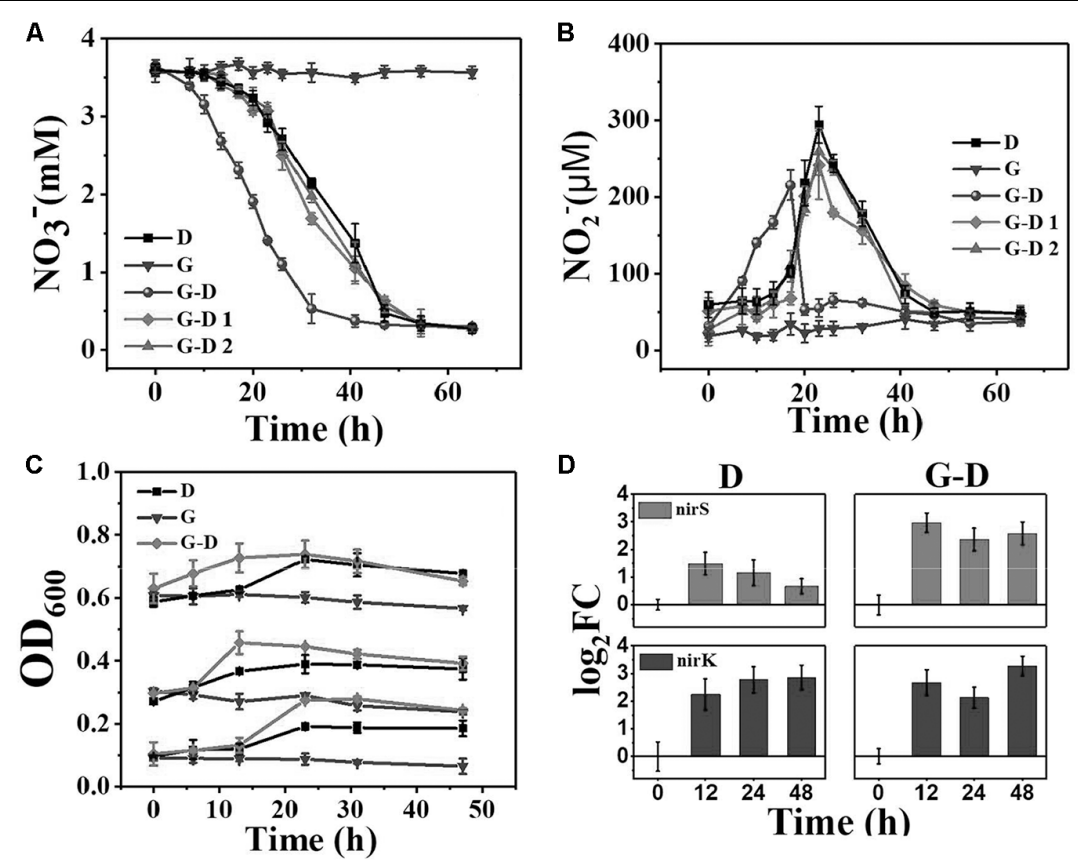

FIGURE 1 | The variations of nitrate $\mathbf{( A )}$ and nitrite $\mathbf{( B )}$ concentrations in $65 \mathrm{~h}$ since the medium change $(t=0)$ at the $\mathrm{C} / \mathrm{N}$ ratio of 6 . D is denitrifying microbial community, G is the pure G. sulfurreducens PCA, G-D is G. sulfurreducens PCA added denitrifying microbial community. G-D 1 and G-D 2 are UV and ultrasonic inactivated $G$. sulfurreducens PCA added denitrifying microbial communities. (C) $\mathrm{OD}_{600}$ variations of groups D, G and G-D with initial values of 0.1, 0.3, and 0.6. Fold changes (D) in nirS, nirk expressions in Groups D and G-D represented as the log2 transformed value. Relative transcript ratios with 16S rRNA as reference were used to determine fold changes as compared to that of the $0 \mathrm{~h}$ sample. Error bars represent standard deviation from three parallel samples.

The pure culture of G. sulfurreducens PCA (Group G) was not able to reduce nitrate to nitrite (Figures 1A,B), showing that all the changes between Groups D and G-D were attributed to the interaction between G. sulfurreducens PCA and other microorganisms. In order to confirm whether the living Geobacter or some components from Geobacter (such as redox cytochromes) mainly resulted in these changes, we mixed G. sulfurreducens PCA treated with ultraviolet radiation (Group G-D1) or ultrasonic fragmentation (Group G-D2) and found that both groups cannot accelerate denitrification (Figure 1A), demonstrated that only the enzymes, proteins or even fragments from G. sulfurreducens cannot accelerate the nitrate removal (Figure 1).

The activities of denitrifying genes were usually evaluated by quantifying the amount of NIR genes (nirK and nirS) using realtime quantitative PCR because the reduction of nitrite is usually the rate-limiting process (Throback et al., 2004). The nirK and nirS encode two types of structurally different but functionally similar NIRs [a copper nitrite reductase encoded by the nirK and a cytochrome cd1-NIR encoded by the nirS (Zumft, 1997)]. Significant increases of nirK and nirS were observed over $12 \mathrm{~h}$ in both Groups of G-D and D (Figure 1D), corresponding to the fast removal of nitrate and nitrite in the initial $48 \mathrm{~h}$. The $\log _{2} \mathrm{FC}$ values of nirK transcripts was constant of $2.70 \pm 0.57$, showing an insignificant change between two groups. However, the $\log _{2} \mathrm{FC}$ values of nirS transcripts gradually decreased from $1.49 \pm 0.40$ to $0.67 \pm 0.28$ from $12 \mathrm{~h}$ to $48 \mathrm{~h}$ in Group D, 2 4 times lower than that of nirK. The addition of G. sulfurreducens
PCA selectively enhanced the expression of nirS to a comparable level of $n i r K$, resulting in a fast and more complete denitrification by avoiding the accumulation of nitrite. This was in accordance with the lower peak of nitrite observed in Group G-D. It should be noted that the genome of G. sulfurreducens PCA does not contain nirK or nirS (Methe et al., 2003), so the enhancement should be due to the change of denitrifying microbial community. The $\mathrm{OD}_{600}$ presented a slight decrease of biomass in Group G, comparing to an obvious increase in the Group D and G-D, showing the growth of bacteria in both groups. Especially, the Group G-D exhibited a faster increase of $\mathrm{OD}_{600}$ in the initial $24 \mathrm{~h}$ than that of Group D, corresponding to the faster acetate and nitrate consumption (Supplementary Figure S4), indicating that the addition of Geobacter accelerated the bacterial growth. In order to have more information of this process, it is necessary to analyze the succession of the microbial community at different time profile.

\section{Reshaping of Denitrifying Microbial Community}

The denitrifying microbial community was initially acclimated at anoxic from activated sludge using methanol as the sole carbon source. According to the records in literatures (Khan and Hiraishi, 2002; Shigematsu et al., 2003; Chistoserdova et al., 2007; Bai et al., 2009), the putative denitrifying bacteria were mainly from genera of Diaphorobacter, Ochrobactrum, Delftia, Shinella, Thermomonas, Pseudomonas, and Methylobacillus (Figure 2). 

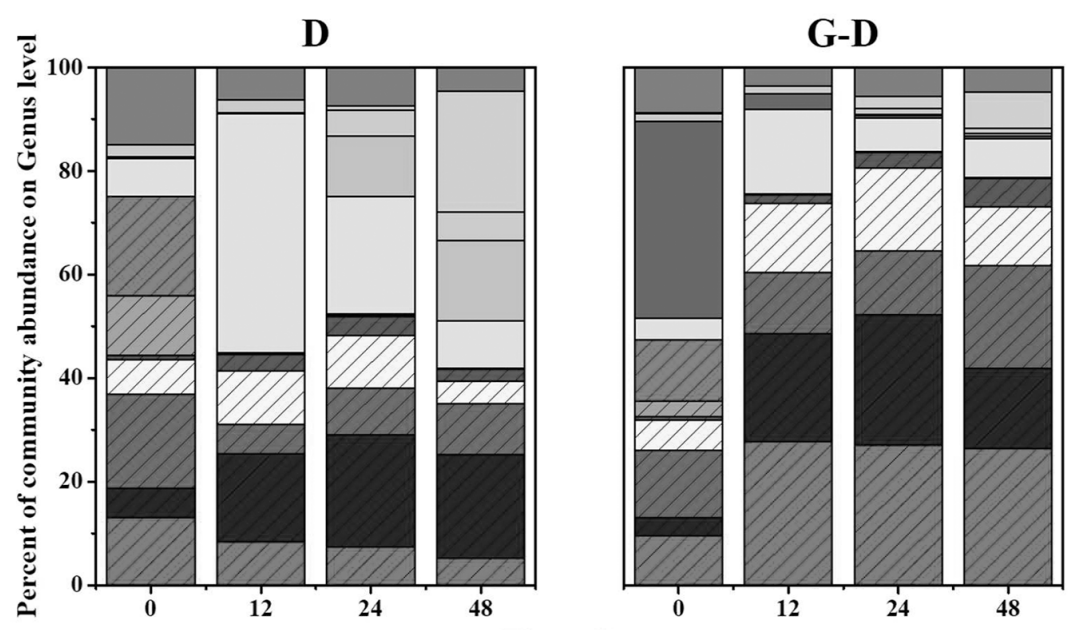

Time (h)
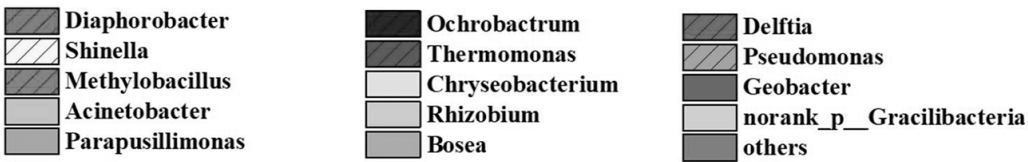

FIGURE 2 | Relative abundance of microbial community at genus level in Groups D (denitrifying microbial community) and G-D (G. sulfurreducens added denitrifying microbial community) at different time profiles. Shaded areas represent the putative denitrifiers. Genera with relative abundance lower than $5 \%$ was classified into group "others." All samples were collected from planktonic cells.
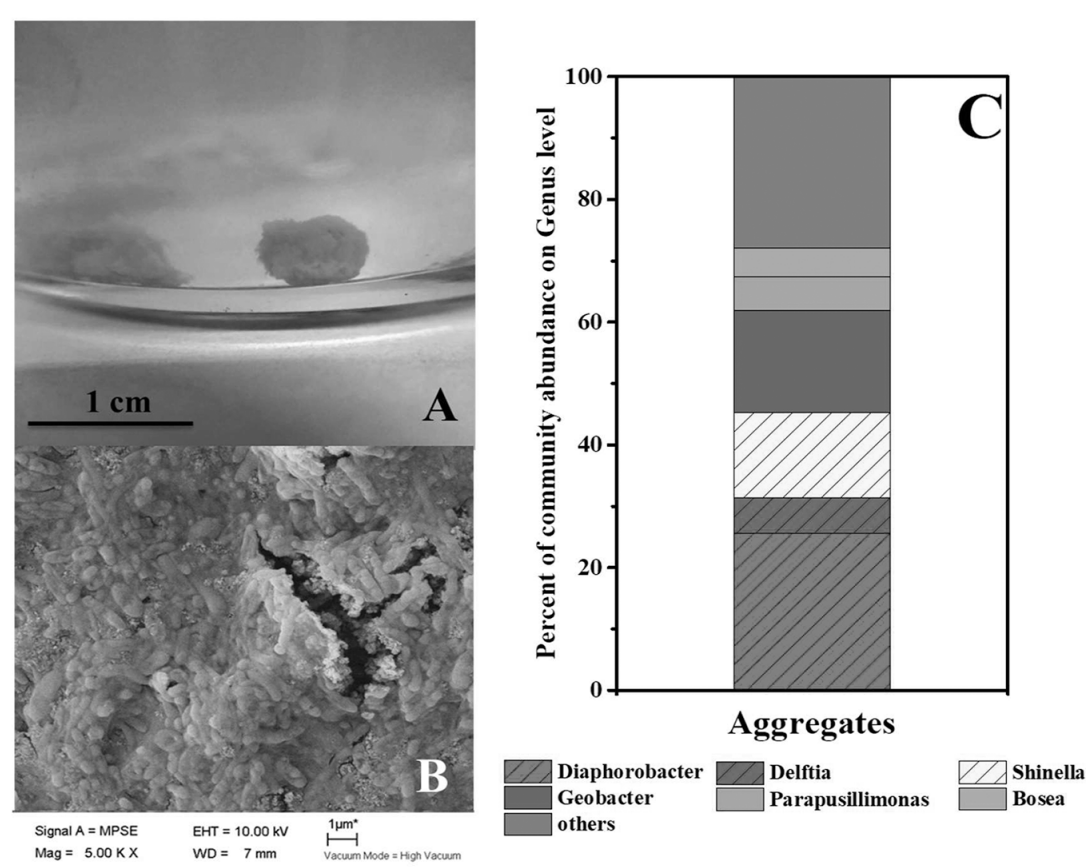

FIGURE 3 | Photo (A) and SEM image (B) of aggregates in G. sulfurreducens PCA added denitrifying microbial community. Relative abundance of microbial community at genus level in the pink aggregates (C).

When it was transferred to anaerobic condition with acetate as the sole carbon source, Pseudomonas and Methylobacillus were replaced by other bacteria, probably because Pseudomonas was aerobic bacteria and Methylobacillus was putative denitrifier specifically living on methanol (Chistoserdova et al., 2007). Some of the genera were selectively enriched after G. sulfurreducens PCA addition. Taking samples at $12 \mathrm{~h}$ as an example, Diaphorobacter predominantly presented (26\%) in Group G-D, 


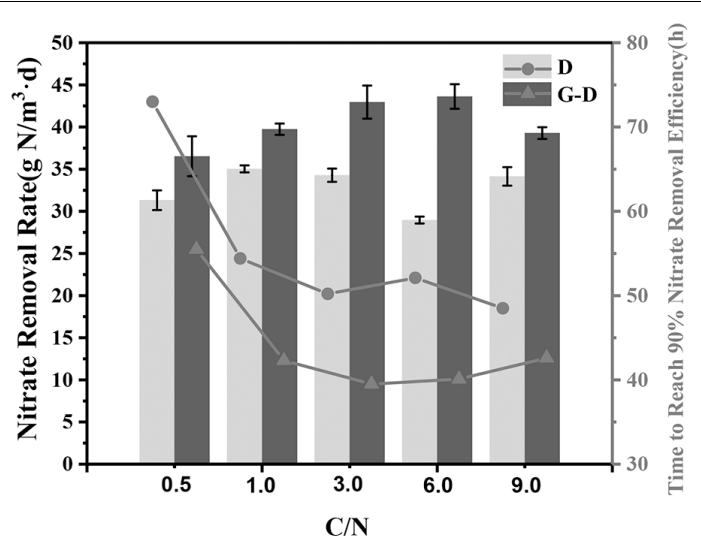

FIGURE 4 | Nitrate removal rate under different $\mathrm{C} / \mathrm{N}$ ratios $(0.5,1,3,6$, and 9) in Group D (denitrifying microbial community) and G-D (G. sulfurreducens added denitrifying microbial community). The rate was calculated by fitting a straight line and taking the derivative for the variations of nitrate removal efficiency. The lines represent the time required to reach $90 \%$ nitrate removal efficiency.

while that genus only accounted for $5.2 \%$ in Group D. Besides, Delftia and Shinella had 20 and 11\% in Group G-D, but their abundances in Group D were relatively small (10 and 4.4\%). There was almost no difference between two conditions in the relative abundance of some putative denitrifiers such as Ochrobactrum and Thermomonas.

On the time scale, Chryseobacterium (a genus of Gramnegative bacteria) in Group D became predominant after $12 \mathrm{~h}$ from $7.2 \%(0 \mathrm{~h})$ to $46 \%$, while the growth of Diaphorobacter and Delftia were inhibited. Two minor genera of Acinetobacter and Gracilibacteria were amplified from $0.1 \%(12 \mathrm{~h})$ to $12 \%(24 \mathrm{~h})$ and $0.8 \%(24 \mathrm{~h})$ to $23 \%(48 \mathrm{~h})$. The microbial community composition was relatively stable after G. sulfurreducens PCA addition (G-D in Figure 2) in $48 \mathrm{~h}$. Different from the Group D, Chryseobacterium were consecutively inhibited in terms of abundance from $16.3 \%$ at $12 \mathrm{~h}$ to $6.5 \%$ at $48 \mathrm{~h}$.

In general, G. sulfurreducens PCA enhanced the total putative denitrifiers from $47 \pm 5 \%$ to $80 \pm 4 \%$. The existence of G. sulfurreducens PCA was beneficial to the growth of putative denitrifiers such as Diaphorobacter, Delftia, and Shinella in the mixed denitrifying microbial community, even though planktonic Geobacter gradually decreased from 38\% (0 h) to $0.4 \%(24 \mathrm{~h})$ in $24 \mathrm{~h}$. It is clear that the syntrophic growth of G. sulfurreducens PCA accelerated the reshaping of microbial community, constructed a stable and efficient (Figure 1) denitrifying community in a couple of its doubling time ( $12 \mathrm{~h}$, the doubling time for Geobacter is $6 \mathrm{~h}$ ). How the Geobacter interact with other microorganisms? Where are they? We observed pink aggregates in Group G-D.

\section{Morphology and Microbial Community of Aggregates}

As mentioned in context, syntrophic partners often gather together to form aggregates. The coculture of G. sulfurreducens and denitrifying microbial community formed large pink aggregates (diameter $>8 \mathrm{~mm}$, Figure $\mathbf{3 A}$ and Supplementary Figure S2) in $24 \mathrm{~h}$, which was different from the aggregates formed in G. sulfurreducens/G. metallireducens cocultures. Rodshape bacterial cells huddled tightly to form a spherical surface, ensuring an effective electron transfer or mass transfer between adjacent cells (Figure 3B). It was showed that the biomass of the aggregates accounted for $45 \%$ of the total biomass (Supplementary Figure S5), showing that bacteria in Group G-D equally distributed between the aggregation and planktonic cells. Sequencing of these aggregates revealed that the Diaphorobacter (26\%), Geobacter (17\%) and Shinella (14\%) were predominant genera, preliminarily demonstrated the syntrophic growth of Geobacter with Diaphorobacter and Shinella (Figure 3C). Comparing to the Geobacter abundance of $0.4 \%$ in suspension, Geobacter mainly accumulated in these aggregates. It could not be ruled out that Delftia was syntrophic partner even though the proportion of Delftia in the community was relatively small (6\%).

The formation of aggregates and the significant acceleration of nitrate removal in cocultures suggested a mechanism of syntrophic interaction where G. sulfurreducens and denitrifiers combine their metabolism to a fast and energy efficient route to utilize acetate and reduce nitrate. It was reported that G. sulfurreducens produce $\mathrm{H}_{2}$ when the electron acceptor is limited (Cord-Ruwish et al., 1998). Therefore, the interspecies electron transfer between Geobacter and denitrifiers in this study probably uses $\mathrm{H}_{2}$ as the electron carrier. Unfortunately, the $\mathrm{H}_{2}$ concentration in the headspace was lower than the detecting limit in all samples, probably because the interspecies hydrogen transfer only allows a very low $(0.02-0.04 \mathrm{~Pa}) \mathrm{H}_{2}$ partial pressure (Cord-Ruwish et al., 1998). DIET might be the other mechanism, which has to be confirmed in binary cultures in the future.

\section{Denitrification at Different C/N Ratios}

In order to test the denitrification performance in this syntrophic system, the $\mathrm{C} / \mathrm{N}$ ratio in the medium was changed to $0.5,1,3$, 6 , and 9 individually. As expected, the nitrate removal rates in all Geobacter added systems were $13 \sim 51 \%$ greater than those in group $\mathrm{D}$ even at a very low $\mathrm{C} / \mathrm{N}$ ratio of 0.5 (Figure 4). All $\mathrm{G}-\mathrm{D}$ groups except for the $\mathrm{C} / \mathrm{N}$ ratio of 0.5 spent $41 \pm 1.5 \mathrm{~h}$ to reach $90 \%$ removal efficiency, but the denitrifying microbial community without Geobacter required $52 \pm 3 \mathrm{~h}$. At the $\mathrm{C} / \mathrm{N}$ ratio of 0.5 , the denitrifying microbial community needed a longer time of $73 \mathrm{~h}$ to reach the same nitrate removal due to the lack of carbon source, while Geobacter shortened the time by $25 \%$ to $56 \mathrm{~h}$. The syntrophic growth of G. sulfurreducens PCA was further demonstrated to accelerate denitrification rate at a broad $\mathrm{C} / \mathrm{N}$ ratio range from 0.5 to 9 .

\section{Implications}

Here we demonstrated that the syntrophic growth of G. sulfurreducens with denitrifying microbial community significantly accelerated the anaerobic denitrification process over a $\mathrm{C} / \mathrm{N}$ ratio range of 0.5 to 9 . Geobacter selectively enriched its potential partners (such as Diaphorobacter, Delftia, and Shinella) in mixed culture to form aggregates, and the expression of nirS was enhanced as a result. Our findings showed the evidence of interspecies electron transfer between exoelectrogens 
and denitrifiers to produce $\mathrm{N}_{2}$, although the exact biological process and scientific mechanism should be further explored. This is very important to understand the nitrogen and metal cycling driven by microorganisms.

Our findings can be further applied to stabilize denitrifying microbial community when the operational conditions changes in wastewaters. For example, AAO, the most widely used technology for denitrification, requires a recirculation of aerobic activated sludge back to anaerobic tank. The denitrification usually has a lag phase due to the change of oxygen and substrate. If a bioelectrochemical system is added to the anaerobic tank in the future, exoelectrogens can accelerate the denitrification process to shorten the hydraulic retention time (HRT) and narrow the volume of the tank, reducing the total cost and energy inputs for denitrification.

\section{AUTHOR CONTRIBUTIONS}

XW designed this experiment and revised the manuscript. YW did most of the test and wrote this manuscript. LZ and SW helped

\section{REFERENCES}

Bai, Y., Sun, Q., Zhao, C., Wen, D., and Tang, X. (2009). Aerobic degradation of pyridine by a new bacterial strain, Shinella zoogloeoides BC026. J. Ind. Microbiol. Biotechnol. 36, 1391-1400. doi: 10.1007/s10295-0090625-629

Boone, D. R., Johnson, R. L., and Liu, Y. (1989). Diffusion of the interspecies electron carriers h2 and formate in methanogenic ecosystems and its implications in the measurement of $\mathrm{km}$ for h2 or formate uptake. Appl. Environ. Microbiol. 55, 1735-1741.

Chistoserdova, L., Lapidus, A., Han, C., Goodwin, L., Saunders, L., Brettin, T., et al. (2007). Genome of Methylobacillus flagellatus, molecular basis for obligate methylotrophy, and polyphyletic origin of methylotrophy. J. Bacteriol. 189, 4020-4027. doi: 10.1128/JB.00045-47

Cord-Ruwish, R., Lovley, D. R., and Schink, B. (1998). Growth of Geobacter sulfurreducens with acetate in syntrophic cooperation with hydrogen-oxidizing anaerobic partners. Appl. Environ. Microbiol. 64, 2232-2236.

Ding, X., Zhao, J., Hu, B., Chen, Y., Ge, G., Li, X., et al. (2016). Mathematical modeling of nitrous oxide production in an anaerobic/oxic/anoxic process. Bioresour. Technol. 222, 39-48. doi: 10.1016/j.biortech.2016. 09.092

Feng, C., Liu, Y., Li, Q., Che, Y., Li, N., and Wang, X. (2016). Quaternary ammonium compound in anolyte without functionalization accelerates the startup of bioelectrochemical systems using real wastewater. Electrochim. Acta 188, 801-808. doi: 10.1016/j.electacta.2015.12.069

Henry, S., Baudoin, E., Lopez-Gutierrez, J. C., Martin-Laurent, F., Brauman, A., and Philippot, L. (2004). Quantification of denitrifying bacteria in soils by nirK gene targeted real-time PCR. J. Microbiol. Methods 59, 327-335. doi: 10.1016/j. mimet.2004.07.002

Ishii, S., Kosaka, T., Hori, K., Hotta, Y., and Watanabe, K. (2005). Coaggregation facilitates interspecies hydrogen transfer between Pelotomaculum thermopropionicum and Methanothermobacter thermautotrophicus. Appl. Environ. Microbiol. 71, 7838-7845. doi: 10.1128/AEM.71.12.7838-7845. 2005

Jackson, B. E., and McInerney, M. J. (2002). Anaerobic microbial metabolism can proceed close to thermodynamic limits. Nature 415, 454-456. doi: 10.1038/ $415454 \mathrm{a}$

Kato, S., Hashimoto, K., and Watanabe, K. (2012). Microbial interspecies electron transfer via electric currents through conductive minerals. Proc. Natl. Acad. Sci. U.S.A. 109, 10042-10046. doi: 10.1073/pnas.1117592109 to grow G. sulfurreducens PCA. CL helped for RNA extraction and sequencing analysis. NL and WL helped to improve the quality of this manuscript.

\section{FUNDING}

This research work was financially supported by National Natural Science Foundation of China (No. 21577068), the Fundamental Research Funds for the Central Universities and 111 Program, Ministry of Education, China (T2017002) and the Tianjin Research Program of Application Foundation and Advanced Technology (15JCYBJC22700).

\section{SUPPLEMENTARY MATERIAL}

The Supplementary Material for this article can be found online at: https://www.frontiersin.org/articles/10.3389/fmicb. 2018.01572/full\#supplementary-material

Khan, S. T., and Hiraishi, A. (2002). Diaphorobacter nitroreducens gen. nov., sp. nov., a poly(3-hydroxybutyrate)-degrading denitrifying bacterium isolated from activated sludge. J. Gen. Appl. Microbiol. 48, 299-308. doi: 10.2323/jgam. 48.299

Lovley, D. R. (2017). Syntrophy goes electric: direct interspecies electron transfer. Annu. Rev. Microbiol. 71, 643-664. doi: 10.1146/annurev-micro-03011702 0420

Lovley, D. R., and Phillips, E. J. (1988). Novel mode of microbial energy metabolism: organic carbon oxidation coupled to dissimilatory reduction of iron or manganese. Appl. Environ. Microbol. 54, 1472-1480.

Methe, B. A., Nelson, K. E., Eisen, J. A., Paulsen, I. T., Nelson, W., Heidelberg, J. F., et al. (2003). Genome of Geobacter sulfurreducens: metal reduction in subsurface environments. Science 302, 1967-1969.

Rotaru, A. E., Shrestha, P. M., Liu, F., Markovaite, B., Chen, S., Nevin, K. P., et al. (2014). Direct interspecies electron transfer between Geobacter metallireducens and Methanosarcina barkeri. Appl. Environ. Microbiol. 80, 4599-4605. doi: 10. 1128/AEM.00895-814

Rotaru, A. E., Shrestha, P. M., Liu, F., Ueki, T., Nevin, K., Summers, Z. M., et al. (2012). Interspecies electron transfer via hydrogen and formate rather than direct electrical connections in cocultures of Pelobacter carbinolicus and Geobacter sulfurreducens. Appl. Environ. Microbiol. 78, 7645-7651. doi: 10. 1128/AEM.01946-1912

Semenec, L., Laloo, A. E., Schulz, B. L., Vergara, I. A., Bond, P. L., and Franks, A. E. (2018). Deciphering the electric code of Geobacter sulfurreducens in cocultures with Pseudomonas aeruginosa via SWATH-MS proteomics. Bioelectrochemistry 119, 150-160. doi: 10.1016/j.bioelechem.2017.09.013

Shigematsu, T., Yumihara, K., Ueda, Y., Numaguchi, M., Morimura, S., and Kida, K. (2003). Delftia tsuruhatensis sp. nov., a terephthalate-assimilating bacterium isolated from activated sludge. Int. J. Syst. Evol. Microbiol. 53(Pt 5), 1479-1483. doi: 10.1099/ijs.0.02285-2280

Srinivasan, V. N., and Butler, C. S. (2017). Ecological and transcriptional responses of anode-respiring communities to nitrate in a microbial fuel cell. Environ. Sci. Technol. 51, 5334-5342. doi: 10.1021/acs.est.6b06572

Stams, A. J., and Plugge, C. M. (2009). Electron transfer in syntrophic communities of anaerobic bacteria and archaea. Nat. Rev. Microbiol. 7, 568-577. doi: 10.1038/ nrmicro2166

Summers, Z. M., Fogarty, H. E., Leang, C., Franks, A. E., Malvankar, N. S., and Lovley, D. R. (2010). Direct exchange of electrons within aggregates of an evolved syntrophic coculture of anaerobic bacteria. Science 330, 1413-1415. doi: $10.1126 /$ science. 1196526 
Thomas, O., and Cerdà, V. (2007). UV-Visible Spectrophotometry of Water and Wastewater. Palma: University of the Balearic Islands.

Throback, I. N., Enwall, K., Jarvis, A., and Hallin, S. (2004). Reassessing PCR primers targeting nirS, nirK and nosZ genes for community surveys of denitrifying bacteria with DGGE. FEMS Microbiol. Ecol. 49, 401-417. doi: 10. 1016/j.femsec.2004.04.011

Yan, X., Han, Y., Li, Q., Sun, J., and Su, X. (2016). Impact of internal recycle ratio on nitrous oxide generation from anaerobic/anoxic/oxic biological nitrogen removal process. Biochem. Eng. J. 106, 11-18. doi: 10.1016/j.bej.2015.11.005

Zumft, W. G. (1997). Cell biology and molecular basis of denitrification. Microbiol. Mol. Biol. Rev. 61, 533-616.
Conflict of Interest Statement: The authors declare that the research was conducted in the absence of any commercial or financial relationships that could be construed as a potential conflict of interest.

Copyright (@) 2018 Wan, Zhou, Wang, Liao, Li, Liu and Wang. This is an open-access article distributed under the terms of the Creative Commons Attribution License (CC BY). The use, distribution or reproduction in other forums is permitted, provided the original author(s) and the copyright owner(s) are credited and that the original publication in this journal is cited, in accordance with accepted academic practice. No use, distribution or reproduction is permitted which does not comply with these terms. 